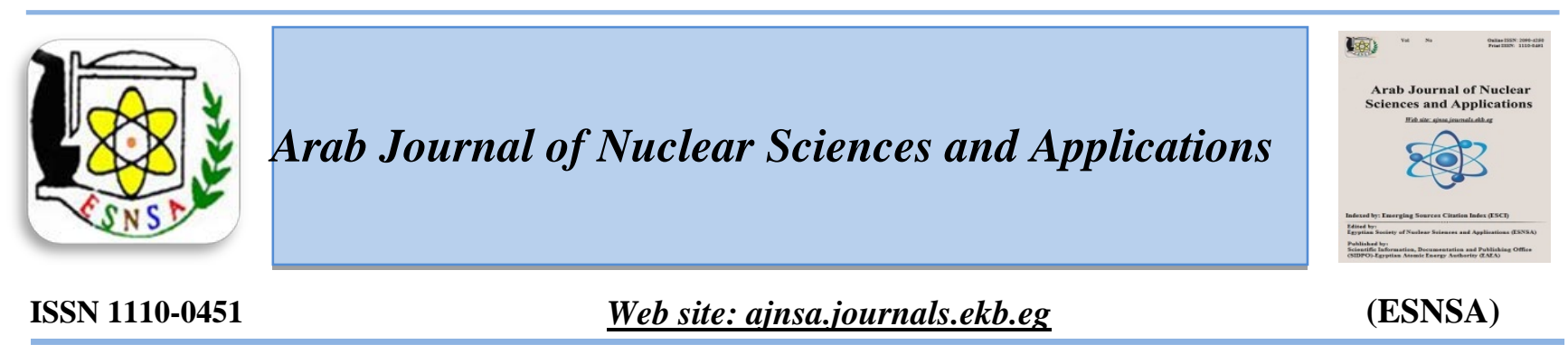

\title{
Green Synthesis of Spherical Gold Nanoparticles by Chitosan for 6 Mercaptopurine Delivery
}

\author{
Amna Hussein ${ }^{(1)}$, Y.A. Badr ${ }^{(1)}$, Samia A. Shouman ${ }^{(2)}$ and Mahmoud A. Sliem ${ }^{(1)}$ \\ ${ }^{1}$ Laser science and interaction, National Institute of Laser Enhanced Sciences, Cairo University \\ ${ }^{2}$ Cancer Biology Department, National Cancer Institute, Cairo University
}

Received $14^{\text {th }}$ Jan. 2018 In the present work, gold nanoparticles were successfully prepared using chitosan as reducing and Accepted $1^{\text {st }}$ Feb. 2018 capping agent and subsequently used as carriers for hydrophobic anticancer 6 mercaptopurine (6 MP). The use of this drug is limited due to its poor bioavailability. The purpose of the present investigation was to evaluate a method for loading hydrophobic drug 6 MP on chitosan reduced gold nanoparticles (AuNPs). The resulting AuNPs and 6MP loaded AuNPs were characterized using ultraviolet visible spectrophotometry and transmission electron microscopy. To estimate the improvement of the drug delivery of AuNPs, breast cancer cells MCF7 were treated with 6 MP and 6 MP loaded AuNPs and the cell viability was studied in vitro using Sulphorhodamine-B (SRB) assay. The data showed that both free 6 MP and 6 MP loaded AuNPs revealed anti-proliferation activity against the cancer cells in dosedependent manners. Moreover, 6 MP loaded AuNPs displays significantly greater anti-proliferation activity than that of free $6 \mathrm{MP}$ at all concentrations and $\mathrm{IC}_{50} 1.93 \mathrm{nM}$. The improvement of antiproliferation activity of 6 MP loaded AuNPs compared to that of 6 MP can be attributed to enhanced intracellular uptake of the functionalized GNPs via the mechanism of endocytosis.

Keywords: Breast cancer/6 Mercaptopurine (6MP)/ Drug delivery/Chitosan / Gold nanoparticles (AuNPs)

\section{Introduction}

Cancer is one of the primary causes of mortality worldwide, the number of new cases of cancer in the world increases each year [1], accounting for approximately 8 million deaths per year [2]. A major problem of the conventional cancer drugs relates to their toxicity which causes the death of healthy cells as well as cancerous cells [3] and acquirement of multidrug resistance (MDR) against anticancer drugs [4]. Although great efforts have been made to overcome MDR, only limited success has been achieved in clinical use[5]. Among various chemotherapeutic agents, 6 Mercaptopurine (6 MP) is one of the most effective immune-suppressants and anti-cancer agents which are widely used to treat inflammatory diseases [6], acute lymphoblastic leukemia and acute myelocytic Leukemia ${ }^{[7]}$. Nanotherapeutics is rapidly progressing and aims to solve the problems of conventional chemotherapy. AuNPs have been used in clinical application as unique drug delivery vehicles due to their distinctive shape, size, and surface-dependent properties [8]. Moreover, their surfaces can be easily functionalized [9]. Chitosan is a $(\beta-1,4)$-linked D-glucosamine, $\mathrm{N}$-deacetylated derivative of chitin constituting the exoskeleton of arthropods and cell walls of fungi and yeast [10]. The unique properties of chitosan make it attractive for many biological applications, including controlled drug release, antibacterial agent and antitumor effect [11] .Chitosan was used

Corresponding author: amna hussein090@yahoo.com

DOI: 10.21608 /ajnsa.2018.2628.1039

(C) Scientific Information, Documentation and Publishing Office (SIDPO)-EAEA 
as a reducing/capping agent in gold nanoparticles production. Chitosan is readily soluble in dilute acidic solutions below $\mathrm{pH}$ 6.0. The solubilisation of chitosan occurs by protonation of the amine group on the $\mathrm{C}-2$ position of the D-glucosamine repeating unit therefore chitosan exhibits polycationic nature possessing metal chelating property which can able to bind metal ions in aqueous solution [12] . Metal chelating chitosan with functional groups, such as amine and hydroxyl groups, can be able to reduce gold precursors in aqueous suspension and also stabilize the formed AuNPs[13] .Chitosan has many advantages, it provides sufficient charge through the amino groups which will help in the subsequent attachment of the biomolecules, optimum stability and consequently helps to improve the uptake of the nanoparticles [14] . In the present work, the use of chitosan as reducing and capping agent in the preparation of AuNPs and subsequently preparation of 6 MP coated chitosan reduced AuNPs by modification of nanoparticles surface with $6 \mathrm{MP}$ as a direct interaction of the drug with nanoparticles are revealed

The progress in the formation of the new composite has been studied via UV-visible, zeta potential and TEM imaging. The obtained results proved that the 6 MP-AuNPs of small size exhibited considerably high toxic MCF 7 compared to the effects of equal doses of free 6 MP.

\section{Materials and Methods}

Chemicals:Tetrachlorauric acid $\left(\mathrm{HAuCl}_{4} \cdot 3 \mathrm{H}_{2} \mathrm{O}\right), 6$ mecaptopurine and chitosan low molecular weight were obtained from Sigma Aldrich Chemical Co., St. Louis, Mo, U.S.A.

Cell lines: Human breast carcinoma cell line (MCF7) was obtained from the American Type Culture Collection (ATCC, MO, USA). The tumor cell line was propagated and maintained by serial sub-culturing in RPMI-164a0 medium containing $10 \%$ FBS and 1\% penicillin/ streptomycin.

Preparation of chitosan reduced gold nanoparticles (AuNPs).

The AuNPs were synthesized using chitosan as a reducing and capping agent. Different chitosan concentrations ( 0.5 and $1 \%$ ) were prepared in $1 \%$ acetic acid to determine the effect of chitosan concentration on the formation of AuNPs.10 $\mu \mathrm{l}$ of
$1.25 \times 10^{-1} \mathrm{M}$ concentrated aqueous solution of chloroauric acid (HAuCl4) was reduced by heating for $15 \mathrm{~min}$ in $10 \mathrm{ml}$ of chitosan solution to yield a ruby-red solution. The formed particle size and shape was investigated using TEM (Transmission Electron Microscope) and their absorption spectrum has been measured using UV-visible spectrophotometer.

\section{Preparation of 6 MP loaded AuNPs}

For loading 6 mecaptopurine on AuNPs, $5 \times 10^{-4} \mathrm{M}$ of $6 \mathrm{MP}$ was mixed with $1 \mathrm{ml}$ of the synthesized AuNPs and sonicated for 10 min until deep red become blue.

Characterization techniques:

$U V$-visible spectroscopy of the prepared nanoparticles

The change in surface plasmon resonance of gold nanoparticles, before and after coating with $6 \mathrm{MP}$ was monitored using double beam spectrophotometer (PG instrument, $\mathrm{T}^{+}$, UK.).200ul from the prepared solutions were diluted to $2 \mathrm{ml}$ with distilled water then placed in $1 \mathrm{~cm}$ UV-quartz and the absorption was recorded within the appropriate scan range $(200 \mathrm{~nm}$ to 800nm).

Nanoparticles morphological determination using TEM imaging

The morphology of the prepared solutions was carried out using TEM - Nanotechnology\& Advanced Material Central Lab. (NAMCL), Agriculture Research Center (ARC). Company name: FEI, Netherland. Model: Tecnai G20, Super twin, double tilt, Applied voltage: $200 \mathrm{kV}$, Magnification Range: up to $1,000,000 \mathrm{X}$ and Gun type: LaB6 Gun. A drop from very dilute solutions were deposited on an amorphous carbon coated copper grid and left to evaporate at room temperature forming a monolayer then detected by TEM

\section{Zeta potential measurements}

The surface charges of gold nanoparticles before and after 6 MP coating were determined by measurement of the zeta potential. The zeta potential was determined using the Zetasizer 300 HAS (Malvern Instruments, Malvern, UK) based on photon correlation spectroscopy. Analysis time was $60 \mathrm{~s}$ and the average zeta potential was determined. The zeta potential of nanoparticulate 
dispersion was determined as such without dilution.

In vitro study for the potential cytotoxicity of $6 \mathrm{MP}$ and 6 MP loaded AuNPs on MCF 7 breast cancer cell line using Sulphorhodamine-B (SRB) assay

This method was carried out in a manner similar to that of Skehan et al. $(199)^{[15]}$. The cells were seeded in 96-well microtiter plates at a concentration of $5 \times 10^{3} \mathrm{Cell} /$ well in a fresh medium and left to attach to the plates for 24 hrs. The cells were incubated with same concentrations of free 6 MP and DOX coated AuNPs (1.25 × 10 $0^{-6}, 2.5 \times 10^{-}$ ${ }^{6}, 5 \times 10^{-6}, 10 \times 10^{-6}, 20 \times 10^{-6} \mathrm{M}$ ) for 48 hrs. The cells were fixed with cold $50 \%$ trichloroacetic acid for 1 $\mathrm{hr}$ at 4 0C and washed with distilled water and stained with $0.4 \%$ SRB. The wells were washed with $1 \%$ acetic acid, air-dried and the dye was solubilized with $10 \mathrm{mM}$ tris base $(\mathrm{pH} 10.5)$ using shaker. The optical density (O.D.) of each well was measured spectrophotometrically at $564 \mathrm{~nm}$ with an ELIZA microplate reader (Meter tech. $\Sigma$ 960, U.S.A.). The mean background absorbance was automatically subtracted and means values of each drug concentration were calculated. The percentage of cell survival was calculated as follows: Survival fraction = O.D (treated cells)/ O.D (control cells). The $\mathrm{IC}_{50}$ values (the concentrations that produced $50 \%$ inhibition of cell growth). The experiment was repeated 3 times independently and each concentration was repeated three times.

\section{Statistical analysis}

Data are expressed as mean \pm SD. Statistical analysis was carried out using Graph Pad Software Prism version 5. The statistical analysis of the transfection assays data was done using Tukey multiple comparison test. Differences were considered statistically significant when $\mathrm{p} \leq 0.01$.

\section{Results}

Characterization of chitosan reduced AuNPs:

As shown in Figure (1), the prepared AuNPs exhibits surface plasmon band (SPB) at $522 \mathrm{~nm}$ which is specific for spherical shape, these results were confirmed by TEM images (Figure 2), the prepared particles have a homogenous spherical shape of a size approximately $15 \pm 5 \mathrm{~nm}$ as shown in Table (1).

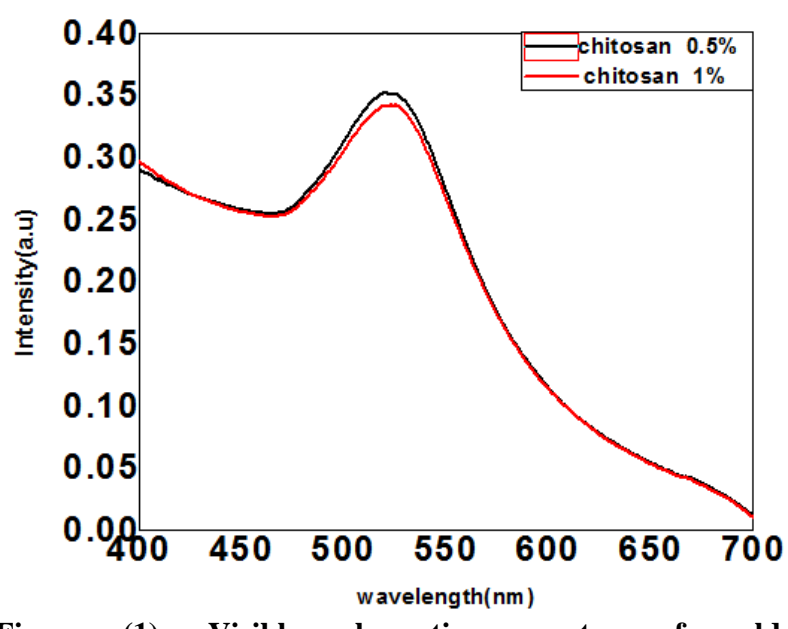

Figure (1): Visible absorption spectra of gold nanoparticles synthesized using various concentrations of chitosan $(0.5 \% \mathrm{w} / \mathrm{v}$ and $1 \% \mathrm{w} / \mathrm{v})$

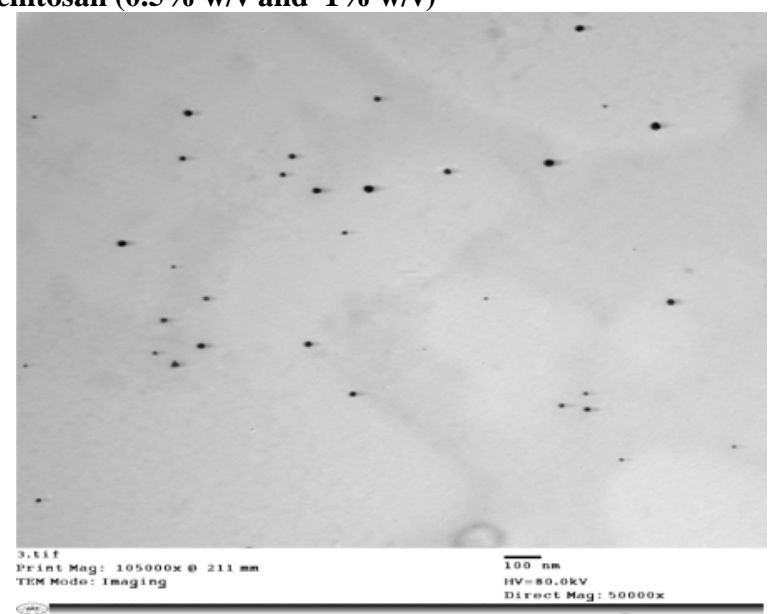

(a)

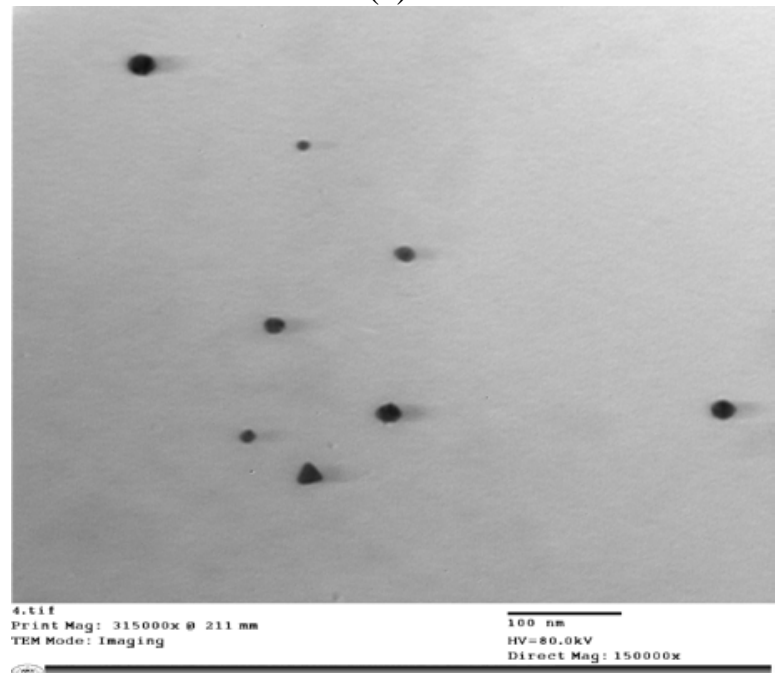

(b)

Figure (2): TEM images of gold nanoparticles reduced using varying concentrations of Chitosan a) $\% 1 \mathrm{w} / \mathrm{v}$ and $b$ ) $0.5 \% \mathrm{w} / \mathrm{v}$

The zeta potential of suspended gold nanoparticles was measured as shown in Figure (4) and Table

Arab J. Nucl. Sci. \& Applic. Vol. 51, No.4 (2018) 
(1), to determine the surface charge and stability of the nanoparticles in aqueous dispersion. The stability of nanoparticles in aqueous is strongly correlated to its zeta potential.

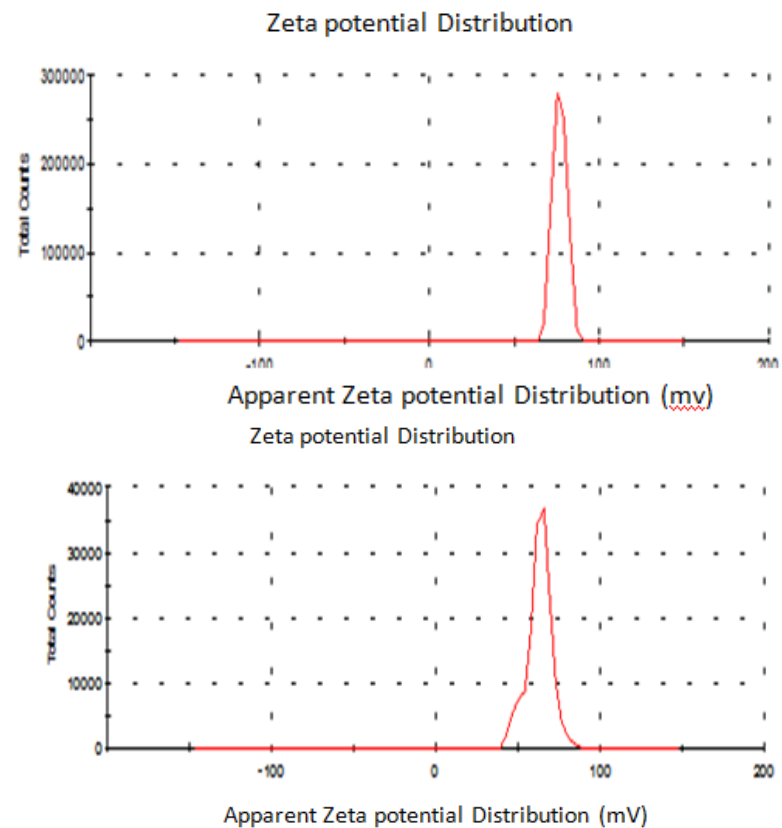

Figure (3): Zeta potential for 1and $0.5 \% \mathrm{~W} / \mathrm{V}$ chitosan reduced gold nanoparticles

Table (1): Average particle size (nm) Zeta potential (mV) and of gold nanoparticles reduced with 1 and $5 \%$ chitosan

\begin{tabular}{|c|c|c|c|}
\hline $\begin{array}{c}\text { Gold } \\
\text { Nanoparticles } \\
\text { reduced } \\
\text { with different } \\
\text { chitosan } \\
\text { concentrations }\end{array}$ & $\begin{array}{c}\text { Average } \\
\text { particle } \\
\text { size(nm) }\end{array}$ & $\begin{array}{c}\text { Zeta } \\
\text { potential } \\
(\mathrm{mV})\end{array}$ & PDI \\
\hline $1 \%$ & $15 \pm 2$ & $76.8 \pm 4.11$ & 0.4 \\
\hline $0.5 \%$ & $15 \pm 5$ & $63 \pm 7.29$ & 0.36 \\
\hline
\end{tabular}

\section{Evaluation of 6 MP loaded chitosan reduced AuNPs:}

The interaction between 6 MP and AuNPs was investigated using UV-visible spectroscopy and the TEM images show an increase in the particle size to $18 \pm n m$ as shown in Figure (5). It could be seen that AuNPs had a characteristic absorption peak at about $522 \mathrm{~nm}$, specific for the plasmon band of the spherical AuNPs. Upon the addition of 6 MP to AuNPs, the absorption spectrum showed a slight red shift in $\lambda$ max of AuNPs plasmon band from $522 \mathrm{~nm}$ to $528 \mathrm{~nm}$ with a decrease in the absorbance intensity due to the dilution. The solution color changed from wine red to blue. At higher wavelengths, another new band was observed around $680 \mathrm{~nm}$.
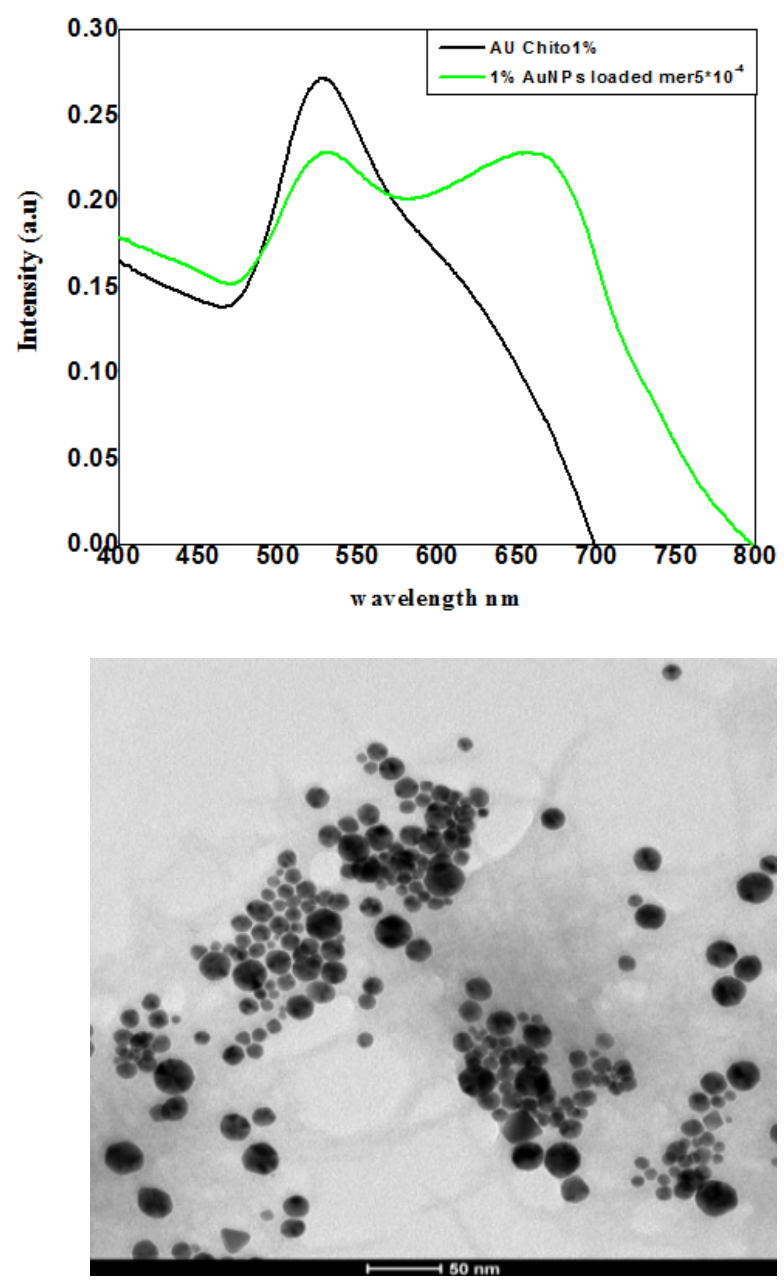

Figure (4): UV-visible spectra of AuNPs and 6 MP loaded AuNPs and TEM images of 6 MP coated 1\% w/v chitosan reduced AuNPs

In vitro cytotoxicity of 6 MP loaded chitosan reduced AuNPs:

Figure (6) reveals the effect of different concentrations of $6 \mathrm{MP}$ and 6MP loaded Chitosan reduced AuNPs (1.25, 2.5, 5, 10 and $20 \mu \mathrm{m})$ on the percentage survival of breast carcinoma cell line (MCF7) after $48 \mathrm{hr}$ exposure to the drug. There was a concentration dependent decrease in cellular proliferation compared to its respective control. It is shown that after 48 hours $6 \mathrm{MP}$ produced a decrease in cell survival of $7 \%$ at $10 \mu \mathrm{m}$ compared to its control, while 6 MP loaded chitosan reduced AuNPs produced a significant decline in the cell survival compared to its control reaching a maximum cytotoxicity of $54 \%$ at $10 \mu \mathrm{m}$. As 
shown in Figure (6), the $\mathrm{IC}_{50}$ value of 6 MP loaded chitosan reduced AuNPs was $1.9 \mu \mathrm{m}$.

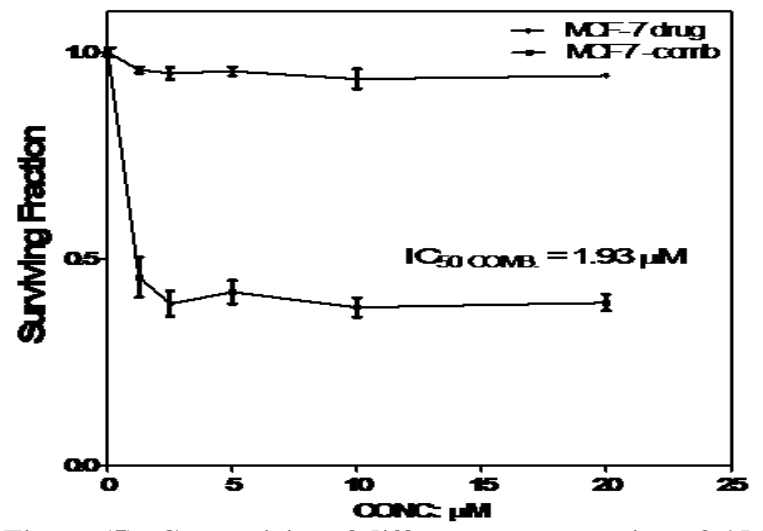

Figure (5): Cytotoxicity of different concentration of 6 MP and 6 MP loaded AuNPs on MCF 7 cell line after 48 hr

\section{Discussion}

Chitosan was used as a reducing and capping agent for eco-friendly synthesis of AuNPs. During the reaction process, there was a color change from dirty yellow to colorless reaching a ruby red color indicating the formation of gold nanoparticles. The reduction of $\mathrm{Au}^{3+}$ (aq) to $\mathrm{Au}^{0}$ follows three electron transfer mechanism ${ }^{[16]}$. As shown in Figure (1), AuNPs exhibits surface Plasmon band SPB at $522 \mathrm{~nm}$ due to collective oscillations of the electron at the surface of the nanoparticles which is associated with the electromagnetic field of the incident light, it can be used to monitor shape, size and aggregation of the nanoparticles ${ }^{[17]}$. The present results showed that $1 \% \mathrm{~W} / \mathrm{V}$ and $0.5 \% \mathrm{~W} / \mathrm{V}$ chitosan concentrations generate a sufficient charge which led to electrostatic stability. The higher the zeta potential, the more stable the nanoparticles due to the stronger repulsive force between each other. The positive charge density of nanoparticles plays an important role in its binding with negatively charged cancer cell membrane ${ }^{[18]}$ .In spite of the application of $6 \mathrm{MP}$ in cancer therapy; purine derivatives exhibit many side effects ${ }^{[19]}$. Loading of 6 MP on the surface of AuNPs is one of the useful methods to overcome the harmful effects of this anticancer drug. There was a red shift in absorption band of AuNPs. This small shift is due to the change in the dielectric constant in the adsorption layer, the increase of the average refractive index of the environment surrounding the nanoparticles and to the increase particles size by the adsorbed layer ${ }^{[20]}$. The appearance of this new band at $680 \mathrm{~nm}$ is due to interparticle interaction between the adjacent gold nanoparticles upon the addition of drug ${ }^{[21]}$.

$6 \mathrm{MP}$ is a prodrug that requires activation to make use of its cytotoxic effect. However, it can be converted into anticancer-active intermediates via enzymatic reaction, which are finally incorporated into the DNA and RNA sequences which in turn trigger cell cycle arrest and apoptosis ${ }^{[22]}$. The cytotoxicity of 6 MP loaded AuNPs may be improved due to better accumulation of drug at its site of action due to targeted delivery. A possible explanation for the activity enhancement of $6 \mathrm{MP}$ loaded AuNPs may be due to the internalization of 6 MP loaded AuNPs by an endocytosis mechanism. Generally, nanoparticles are nonspecifically internalized into cells via endocytosis or phagocytosis compared to the passive diffusion mechanism of free drugs into cells ${ }^{[23]}$.

\section{Conclusions}

The present work demonstrated a method for the biosynthesis of spherical gold nanoparticles using a biocompatible polymer chitosan with improved surface properties for binding of biomolecules for the anticancer drug $6 \mathrm{MP}$. Both free 6MPand $6 \mathrm{MP}$ loaded AuNPs were examined and compared for their anti-proliferation activities against MCF-7 breast cancer cell line. The results showed that GNPs significantly enhance the anti-proliferation activity of 6 MP which can be the result of enhanced intracellular uptake of this drug by endocytosis mechanism. Therefore, the improved anti-proliferation activity of $6 \mathrm{MP}$ by AuNPs carriers could make possible the reduction of the overall concentration of the drug and its side effects during cancer treatment.

\section{References}

1.Ginsberg G, Lim S, Lauer J, Johns B, Sepulveda C, (2010) Prevention, screening and treatment of colorectal cancer: a global and regional generalized cost effectiveness analysis. Cost EffResour Alloc,8: 2-17.

2.Torre LA, Bray F, Siegel RL, Ferlay J, Lortet-Tieulent J, Jemal A,(2015) Global cancer statistics, CA Cancer J Clin ; 65: 87-108.

3.Rozenberg M, Shoham G. (2007) FTIR spectra of solid poly-Llysine in the stretching $\mathrm{NH}$ mode range. Biophys Chem;125:166-171.

4.Gottesman MM ,Fojo, T; Bates, SE,(2002) Multidrug resistance in cancer: Role of ATP-dependent transporters. Nat. Rev. Cancer , 2, 48-58. 
5.Szakacs, G; Paterson, JK; Ludwig, JA; Genthe, CB; Gottesman, MM,(2006)Targeting multidrug resistance in cancer. Nat. Rev. Drug Discov. , 5, 219234.

6.Cuin, A, Massabni, AC, Pereira, GA, Leite, CQF, Pavan, FR, Costa, RS, Heinrich, TA, Costa-Neto, CM (2011) 6-mercaptopurine complexes with silver and gold ions: Anti-tuberculosis and anti-cancer activities.. Biomedicine and Pharmacotherapy 65: 334-338.

7.Wang, T, Liu, X, CHEN, P, (2013) A sensitive inhibition chemiluminescence method for the determination of 6-mercaptopurine in tablet and biological fluid using the reaction of luminol-Ag (III) complex in alkaline medium Journal of Luminescence 134: 154-159.

8. Ghosh, P; Han, G; De, M; Kim, CK; Rotello, VM, (2008) Gold nanoparticles in delivery applications. Adv. Drug Deliv. Rev. , 60, 1307-1315.

9.Shan, J.; Tenhu, H.(2007) Recent advances in polymer protected gold nanoparticles: Synthesis, properties and applications. Chem. Commun. , 44, 4580-4598.

10.RinaudoM, (2006) Chitin and chitosan: Properties and applications. Prog. Polym. Sci. 31 603-632

11.M.N.V.Ravi Kumar, R.A.A.Muzzarelli, C.Muzzarelli, H.Sashiwa, A.J.Domb.(2004) Chitosan Chemistry and Pharmaceutical Perspectives. Chem. Rev. 104 6017- 6084

12.D Wei, Y Ye, $\mathrm{X}$ Jia, C Yuan, W Qian, (2010)Chitosan as an active support for assembly of metal nanoparticles and application of the resultant bioconjugates in catalysis, Carbohydr. Res. 345

13.D Wei, Y Ye, X Jia, C Yuan, W Qian, (2010)Chitosan as an active support for assembly of metal nanoparticles and application of the resultant bioconjugates in catalysis, Carbohydr. Res. 345

14.H Huang, X Yang, (2004) Synthesis of chitosanstabilized gold nanoparticles in the absence/presence of tripolyphosphate, Biomacromolecules 5 23402346

15.Devika R. Bhumkar, Hrushikesh M. Joshi, Murali Sastry, and Varsha B. Pokharkar(2007) Chitosan Reduced Gold Nanoparticles as Novel Carriers for Transmucosal Delivery of Insulin. Pharmaceutical Research, Vol. 24, No. 8,

16.Skehan P, Storeng R, Scudiero D, Monks A, McMahon VD, Warren JT, Bokesch H, Kenney S and Boyd MR(1990|).New colorimetric cytotoxicity assay for anticancer-drug screening. J. Natl. Cancer Inst.,; 82: 1107.

17.M Brust, M Walker, D Bethell, DJ Schiffrin, RJ Whyman, (1994) Synthesis of thiolderivatised gold nanoparticles in a two-phase Liquid-Liquid system, J. Chem. Soc., Chem Commun 801-802.

18.Grabar KC, Freeman RG, Hommer MB, Natan MJ (1995) Preparation and characterization of Au colloid monolayers. Anal Chem 67:735-743
19.Sun, Y,\&Wan, A J ,(2007)Preparation of nanoparticles composed of chitosan and its derivatives as delivery systems for macromolecules. Journal of Applied Polymer Science, 105, 552-561.

20.Wenz HH, Ho"genauer C, Fickert P, Petritsch W (2004) Thioguanineinduced symptomatic thrombocytopenia. Am J Gastroenterol99:1195

21.Tom RT, Suryanarayanan V, Reddy GP, Baskaran S and Pradeep T, (2004) Ciprofloxacin protected gold nanoparticles. Langmuir, (20):1909-1914.

22.Swann PF et al (1996) Role of postreplicative DNA mismatch repair in the cytotoxic action of thioguanine. Science273:1109-1111

23.Sadaf Aghevlian , Reza Yousefi , Reza Faghihi • Abdolkarim Abbaspour , Ali Niazi Mansooreh Jaberipour Ahmad Hosseini (2012)The improvement of anti-proliferation activity against breast cancer cell line of thioguanine by gold Nanoparticles Med Chem Res DOI 10.1007/s00044-012-0030-12 